\title{
Front Matter: Volume 7737
}

, "Front Matter: Volume 7737," Proc. SPIE 7737, Observatory Operations: Strategies, Processes, and Systems III, 773701 (6 October 2010); doi: 10.1117/12.869085

SDIE Event: SPIE Astronomical Telescopes + Instrumentation, 2010, San Diego, SPIE. California, United States 


\title{
PROCEEDINGS OF SPIE
}

\section{Observatory Operations: Strategies, Processes, and Systems III}

\author{
David R. Silva \\ Alison B. Peck \\ B. Thomas Soifer \\ Editors
}

30 June-2 July 2010

San Diego, California, United States

Sponsored by

SPIE

Cooperating Organizations

American Astronomical Society (United States) • Association of Universities for Research in Astronomy, Inc. (United States) • Astronomical Society of Japan (Japan) • Atacama Large Millimeter/Submillimeter Array • Ball Aerospace \& Technologies Corporation (United States) • CASCA-Canadian Astronomical Society (Canada) • Commissariat à l'Energie Atomique (France) • European Astronomical Society (Switzerland) - European Organisation for Astronomical Research in the Southern Hemisphere (Germany) Japan Aerospace Exploration Agency (Japan) - Jet Propulsion Laboratory (United States) • NASA Goddard Space Flight Center (United States) • National Astronomical Observatory Japan (Japan) National Radio Astronomy Observatory • SOFIA-Stratospheric Observatory for Infrared Astronomy (United States) • Thirty Meter Telescope Project (United States) • W. M. Keck Observatory (United States)

Published by

SPIE

Volume 7737 
The papers included in this volume were part of the technical conference cited on the cover and title page. Papers were selected and subject to review by the editors and conference program committee. Some conference presentations may not be available for publication. The papers published in these proceedings reflect the work and thoughts of the authors and are published herein as submitted. The publisher is not responsible for the validity of the information or for any outcomes resulting from reliance thereon.

Please use the following format to cite material from this book:

Author(s), "Title of Paper," in Observatory Operations: Strategies, Processes, and Systems III, edited by David R. Silva, Alison B. Peck, B. Thomas Soifer, Proceedings of SPIE Vol. 7737 (SPIE, Bellingham, WA, 2010) Article CID Number.

ISSN 0277-786X

ISBN 9780819482273

Published by

SPIE

P.O. Box 10, Bellingham, Washington 98227-0010 USA

Telephone +1 3606763290 (Pacific Time) · Fax +1 3606471445

SPIE.org

Copyright (C) 2010, Society of Photo-Optical Instrumentation Engineers

Copying of material in this book for internal or personal use, or for the internal or personal use of specific clients, beyond the fair use provisions granted by the U.S. Copyright Law is authorized by SPIE subject to payment of copying fees. The Transactional Reporting Service base fee for this volume is $\$ 18.00$ per article (or portion thereof), which should be paid directly to the Copyright Clearance Center (CCC), 222 Rosewood Drive, Danvers, MA 01923. Payment may also be made electronically through CCC Online at copyright.com. Other copying for republication, resale, advertising or promotion, or any form of systematic or multiple reproduction of any material in this book is prohibited except with permission in writing from the publisher. The CCC fee code is 0277-786X/10/\$18.00.

Printed in the United States of America.

Publication of record for individual papers is online in the SPIE Digital Library.

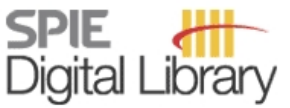

SPIEDigitalLibrary.org

Paper Numbering: Proceedings of SPIE follow an e-First publication model, with papers published first online and then in print and on CD-ROM. Papers are published as they are submitted and meet publication criteria. A unique, consistent, permanent citation identifier (CID) number is assigned to each article at the time of the first publication. Utilization of CIDs allows articles to be fully citable as soon they are published online, and connects the same identifier to all online, print, and electronic versions of the publication. SPIE uses a six-digit CID article numbering system in which:

- The first four digits correspond to the SPIE volume number.

- The last two digits indicate publication order within the volume using a Base 36 numbering system employing both numerals and letters. These two-number sets start with 00, 01, 02, 03, 04, $05,06,07,08,09,0 A, 0 B \ldots 0 Z$, followed by 10-1Z, 20-2Z, etc.

The CID number appears on each page of the manuscript. The complete citation is used on the first page, and an abbreviated version on subsequent pages. Numbers in the index correspond to the last two digits of the six-digit CID number. 


\section{Contents}

xi Conference Committee

xiii Unknowns and unknown unknowns: from dark sky to dark matter and dark energy

(Plenary Paper) [7733-501]

Y. Suto, The Univ. of Tokyo (Japan)

xxv Optical synoptic telescopes: new science frontiers (Plenary Paper) [7733-502]

J. A. Tyson, Univ. of California, Davis (United States)

\section{SESSION 1 SITE AND FACILITY OPERATIONS I}

773705 Constructing the EVLA while operating the VLA [7737-04]

R. Dickman, M. McKinnon, C. Chandler, R. Perley, M. Rupen, J. McMullin, B. Butler, B. Clark, K. Sowinski, National Radio Astronomy Observatory (United States); J. Ulvestad, National Radio Astronomy Observatory (United States) and National Science Foundation (United States)

773706 Mixing completion, commissioning, and operations at the LBT [7737-05]

R. F. Green, J. M. Hill, J. Brynnel, J. H. Slagle, D. S. Ashby, N. J. Cushing, J. K. Little,

R. M. Wagner, M. Pedani, Large Binocular Telescope Observatory (United States)

773707 A new La Silla site operations paradigm [7737-06]

G. Ihle, B. Ahumada, J. Duk, J. C. Fluxá, A. González, I. Kastinen, A. Kaufer, E. Matamoros, M. Pavez, J. C. Pineda, A. Pizarro, I. Saviane, P. Sinclaire, M. Sterzik, European Southern Observatory (Chile)

773708 APEX: five years of operations [7737-08]

A. Lundgren, D. Rabanus, European Southern Observatory (Chile); R. Güsten, K. Menten, Max-Planck-Institut für Radioastronomie (Germany); T. de Zeeuw, European Southern Observatory (Germany); H. Olofsson, Onsala Space Observatory, Chalmers Univ. of Technology (Sweden); A. Kaufer, L.-Å. Nyman, European Southern Observatory (Chile); P. Bergman, Onsala Space Observatory, Chalmers Univ. of Technology (Sweden); C. de Breuck, European Southern Observatory (Germany); F. Wyrowski, Max-Planck-Institut für Radioastronomie (Germany); C. Agurto, F. Azagra, M. Dumke, F. Mac-Auliffe, M. Martinez, F. Montenegro, European Southern Observatory (Chile); D. Muders, Max-Planck-Institut für Radioastronomie (Germany); V. Reveret, CEA, IRFU/SAp, Lab. AIM, CNRS (France); C. Risacher, SRON (Netherlands); R. Parra, G. Siringo, G. Wieching, European Southern Observatory (Chile)

773709 Laser Guide Star operations at the Gemini North Observatory [7737-09]

A. C. Matulonis, Gemini North Observatory (United States) 
7737 OA Laser operations at the 8-10m class telescopes Gemini, Keck, and the VLT: lessons learned, old and new challenges [7737-10]

P. Amico, European Southern Observatory (Germany); R. D. Campbell, W. M. Keck

Observatory (United States); J. C. Christou, Gemini Observatory (United States)

\section{SESSION 2 SITE AND FACILITY OPERATIONS II}

7737 OD Testing and validation of orbital operations plans for the MESSENGER mission [7737-13] A. F. Berman, The Johns Hopkins Univ. (United States); D. L. Domingue, Planetary Science Institute (United States); M. E. Holdridge, T. H. Choo, R. J. Steele, R. G. Shelton, The Johns Hopkins Univ. (United States)

$7737 \mathrm{OE} \quad$ Using the Baldrige criteria for observatory strategic and operations planning [7737-14] N. M. Radziwill, James Madison Univ. (United States) and National Radio Astronomy Observatory (United States); L. Mitchell, National Radio Astronomy Observatory (United States)

\section{SESSION 3 SCIENCE OPERATIONS PROCESSES}

7737 OG ALMA science operations [7737-16]

L.-Å. Nyman, European Southern Observatory (Chile); P. Andreani, European Southern Observatory (Germany); J. Hibbard, National Radio Astronomy Observatory (United States);

S. K. Okumura, National Astronomical Observatory of Japan (Japan)

$7737 \mathrm{OH} \quad$ Kepler Science Operations processes, procedures, and tools [7737-17]

J. R. Hall, K. Ibrahim, T. C. Klaus, Orbital Sciences Corp. (United States) and NASA Ames Research Ctr. (United States); M. T. Cote, NASA Ames Research Ctr. (United States); C. Middour, Orbital Sciences Corp. (United States) and NASA Ames Research Ctr. (United States); M. R. Haas, J. L. Dotson, NASA Ames Research Ctr. (United States); B. Stroozas, Stroozas FlightOps (United States); M. WU, Bastion Technologies (United States) and NASA Ames Research Ctr. (United States); J. Sommers, Orbital Sciences Corp. (United States) and NASA Ames Research Ctr. (United States); P. Bhavsar, NASA Ames Research Ctr. (United States)

$7737 \mathrm{OI}$ The care and feeding of the JWST on-board event-driven system [7737-18] V. Balzano, Space Telescope Science Institute (United States); D. Zak, Computer Sciences Corp. (United States); W. Whitman, Space Telescope Science Institute (United States)

7737 0J Gemini Observatory: five years of multi-instrument queve operations [7737-19] I. Jorgensen, Gemini Observatory (United States); B. Rodgers, Gemini Observatory (Chile); D. R. Crabtree, Gemini Observatory (Chile) and Hertzberg Institute of Astrophysics (Canada)

7737 OL Downsizing a great observatory: reinventing Spitzer in the warm mission [7737-21] L. J. Storrie-Lombardi, Spitzer Science Ctr., California Institute of Technology (United States); S. R. Dodd, Jet Propulsion Lab. (United States) 
7737 OM The VLT rapid-response mode: implementation and scientific results [7737-22]

P. M. Vreeswijk, Univ. of Copenhagen (Denmark) and Univ. of Iceland (Iceland); A. Kaufer, European Southern Observatory (Chile); J. Spyromilio, European Southern Observatory (Germany); R. Schmutzer, C. Ledoux, A. Smette, European Southern Observatory (Chile); A. De Cia, Univ. of Iceland (Iceland)

7737 ON Managing target of opportunity (ToO) observations in queve mode at Gemini Observatory [7737-23]

K. C. Roth, Gemini North Observatory (United States); E. R. Carrasco, B. W. Miller, Gemini South Observatory (Chile); A. W. Stephens, I. Jørgensen, Gemini North Observatory (United States); B. Rodgers, Gemini South Observatory (Chile)

773700 LCOGT sites and site operations [7737-24]

J. J. Martinez, T. M. Brown, P. Conway, M. Elphick, M. Falarski, E. Hawkins, W. Rosing,

J. Shobbrook, Las Cumbres Observatory Global Telescope Network (United States)

7737 OP Scheduling observations on the LCOGT network [7737-25]

E. Hawkins, N. Baliber, M. Bowman, T. Brown, B. Burleson, S. Foale, M. Ford, T. Lister, M. Norbury, E. Saunders, Z. Walker, Las Cumbres Observatory Global Telescope Network (United States)

\section{SESSION 5 TRANSIENT EVENTS AND OBSERVATORY OPERATIONS}

7737 OV Transient alert operations for the Large Synoptic Survey Telescope [7737-32]

R. C. Smith, R. Seaman, National Optical Astronomy Observatory (United States); J. Kantor, T. Axelrod, LSST Corp. (United States)

\section{SESSION 6 DYNAMIC OBSERVATORY SCHEDULING}

7737 OW New observing concepts for ESO survey telescopes [7737-33]

T. Bierwirth, European Southern Observatory (Germany); T. Szeifert, European Southern Observatory (Chile); D. Dorigo, P. Nunes, M. Rejkuba, European Southern Observatory (Germany); K. Baugh, Michael Bailey Associates GmbH (Germany) and European Southern Observatory (Germany); M. Klein Gebbinck, European Southern Observatory (Germany); A. Manning, Michael Bailey Associates GmbH (Germany) and European Southern Observatory (Germany); D. Muravov, Gesellschaft für Technische Kommunikation (Germany) and European Southern Observatory (Germany); I. Vera, European Southern Observatory (Germany)

7737 0X Partner time sharing at the Submillimeter Array [7737-34] G. Petitpas, Q. Zhang, C. Katz, N. Patel, R. Blundell, Harvard-Smithsonian Ctr. for Astrophysics (United States)

7737 OY JWST planning and scheduling operations and concepts [7737-35] W. M. Kinzel, Computer Sciences Corp. (United States) and Space Telescope Science Institute (United States) 
$7737 \mathrm{OZ}$ Simulation of autonomous observing with a ground-based telescope: the LSST experience [7737-36]

S. Ridgway, National Optical Astronomy Observatory (United States); K. Cook, Lawrence Livermore National Lab. (United States) and LSST Corp. (United States); M. Miller, National Optical Astronomy Observatory (United States); C. Petry, Steward Observatory, The Univ. of Arizona (United States); S. Chandrasekharan, A. Saha, National Optical Astronomy Observatory (United States); R. Allsman, LSST Corp. (United States); T. Axelrod, Steward Observatory, The Univ. of Arizona (United States); C. Claver, F. Delgado, National Optical Astronomy Observatory (United States); Z. Ivezic, L. Jones, S. Krughoff, Univ. of Washington (United States); F. Pierfederici, Harvard-Smithsonian Ctr. for Astrophysics (United States); P. Pinto, Steward Observatory, The Univ. of Arizona (United States)

\section{SESSION 7 REMOTE ROBOTIC AND SERVICE}

773711 Switching the Liverpool Telescope from a full-service operating model to self-service [7737-38]

R. J. Smith, N. R. Clay, S. N. Fraser, J. M. Marchant, C. M. Moss, I. A. Steele, Liverpool John Moores Univ. (United Kingdom)

773712 A shared approach to supporting remote observing for multiple observatories [7737-39] R. I. Kibrick, Lick Observatory, Univ. of California, Santa Cruz (United States); G. D. Wirth, W. M. Keck Observatory (United States); E. L. Gates, B. J. Grigsby, W. T. S. Deich, K. Lanclos, S. L. Allen, Lick Observatory, Univ. of California, Santa Cruz (United States)

773715 Mopra remote observing: a story of innovation and success [7737-93]

B. T. Indermuehle, P. G. Edwards, CSIRO Astronomy and Space Science (Australia)

\section{SESSION 8 ARCHIVE OPERATIONS AND LEGACY}

773716 Spitzer Heritage Archive [7737-43]

X. Wu, T. Roby, L. Ly, IRSA/SSC, California Institute of Technology (United States)

773718 Science data production at ESO: strategy, plans, and lessons learned [7737-45] M. Romaniello, W. Freudling, European Southern Observatory (Germany); A. Smette, C. Dumas, European Southern Observatory (Chile); P. Ballester, European Southern Observatory (Germany)

\section{SESSION 9 USER SUPPORT}

773719 User support: new ways forward after 10 years of successful VLT operations [7737-48] F. Primas, European Southern Observatory (Germany)

7737 IA ALMA science operations and user support: software [7737-49] M. G. Rawlings, L.-Å. Nyman, B. Vila Vilaro, Joint Atacama Large Millimeter Array Observatory (Chile) 
7737 IB Handling observation proposals for SALT [7737-50]

C. Hettlage, D. A. H. Buckley, A. C. Charles, South African Astronomical Observatory (South Africa); M. Cordiner, NASA Goddard Space Flight Ctr. (United States); D. R. Harbeck, National Optical Astronomy Observatory (United States); T.-O. Husser, Georg-August-Univ. Göttingen (Germany); K. H. Nordsieck, J. W. Percival, Univ. of Wisconsin-Madison Space Astronomy Lab. (United States); E. Romero Colmenero, South African Astronomical Observatory (South Africa); M. D. Still, NASA Ames Research Ctr. (United States)

7737 1C SMARTS revealed [7737-80]

J. P. Subasavage, Cerro Tololo Inter-American Observatory (Chile); C. D. Bailyn, Yale Univ. (United States); R. C. Smith, Cerro Tololo Inter-American Observatory (Chile); T. J. Henry, Georgia State Univ. (United States); F. M. Walter, Stony Brook Univ. (United States); M. M. Buxton, Yale Univ. (United States)

\section{SESSION 10 OPERATIONS AND DATA QUALITY CONTROL}

7737 ID Calibration of the LSST instrumental and atmospheric photometric passbands [7737-52] D. L. Burke, SLAC National Accelerator Lab. (United States); T. Axelrod, Steward Observatory, Univ. of Arizona (United States); A. Barrau, S. Baumont, Lab. de Physique Subatomique et de Cosmologie de Grenoble, CNRS-IN2P3 (France); S. Blondin, Ctr. de Physique des Particules de Marseille, CNRS-IN2P3 (France); C. Claver, National Optical Astronomy Observatory (United States); A. Gorecki, Lab. de Physique Subatomique et de Cosmologie de Grenoble, CNRS-IN2P3 (France); Ž. Ivezić, L. Jones, Univ. of Washington (United States);

V. Krabbendam, M. Liang, A. Saha, National Optical Astronomy Observatory (United States); A. Smith, Austin Peay State Univ. (United States); R. C. Smith, Cerro Tololo Inter-American Observatory (United States); C. W. Stubbs, Harvard-Smithsonian Ctr. for Astrophysics, Harvard Univ. (United States); C. Vescovi, Lab. de Physique Subatomique et de Cosmologie de Grenoble, CNRS-IN2P3 (France); LSST Project Team, LSST Corp. (United States)

$7737 \mathrm{IF} \quad$ Solving the global photometric self-calibration problem in LSST [7737-54]

R. L. Jones, Univ. of Washington (United States); N. Padmanabhan, Yale Univ. (United States); Ž. Ivezić, Univ. of Washington (United States); T. Axelrod, Steward Observatory, The Univ. of Arizona (United States); J. Bartlett, Lab. d'AstroParticule et Cosmologie, Univ. Paris Diderot (United States); D. Burke, SLAC National Accelerator Lab. (United States); D. Cinabro, Wayne State Univ. (United States); M. Crézé, Lab. d'AstroParticule et Cosmologie, Univ. Paris Diderot (United States); B. Popescu, Univ. of Cincinnati (United States); A. Saha, National Optical Astronomy Observatory (United States)

7737 IG The physical model in action: quality control for X-Shooter [7737-56] S. Moehler, P. Bristow, F. Kerber, A. Modigliani, J. Vernet, European Southern Observatory (Germany)

7737 1H Quality control and data flow operations of the survey instrument VIRCAM [7737-55] W. Hummel, R. Hanuschik, European Southern Observatory (Germany); L. de Bilbao, European Southern Observatory (Germany), Fundación Española para la Ciencia y la Tecnología (Spain), and Ctr. Superior de Investigaciones Científicas (Spain); S. Mieske, T. Szeifert, V. Ivanov, European Southern Observatory (Chile); S. Castro, European Southern Observatory (Germany) 
7737 II Handling heterogeneous arrays: calibrations and data reduction [7737-57]

S. A. Corder, National Radio Astronomy Observatory, Joint Atacama Large Millimeter Submillimeter Array Observatory (Chile); M. C. H. Wright, Univ. of California, Berkeley (United States); J. Koda, Stony Brook Univ. (United States)

$77371 \mathrm{~J}$ The APEX calibration plan: goals, implementation, and achievements [7737-58] M. Dumke, F. Mac-Auliffe, European Southern Observatory (Chile)

\section{POSTER SESSION}

7737 1L Preventive maintenance optimization at Paranal Observatory [7737-60]

E. F. Bugueno, European Southern Observatory (Chile)

7737 1M Reliability culture at La Silla Paranal Observatory [7737-61]

S. Gonzalez, European Southern Observatory (Chile)

7737 IN Data management subsystem software architecture for JWST [7737-62]

D. A. Swade, Space Telescope Science Institute (United States)

773710 Queve observing at the Observatoire du Mont-Mégantic 1.6-m telescope [7737-63]

É. Artigau, R. Lamontagne, R. Doyon, L. Malo, Univ. de Montréal (Canada) and Observatoire du Mont-Mégantic (Canada)

7737 IR Recent developments for the SINFONI pipeline [7737-66]

K. Mirny, Radius ZUG (Germany); A. Modigliani, M. J. Neeser, European Southern

Observatory (Germany); D. Nürnberger, European Southern Observatory (Chile)

7737 is The GPS water vapor monitor and thermal astronomy at Gemini South [7737-67]

J. Radomski, G. Trancho, L. Fuhrman, Gemini Observatory (Chile); M. Falvey, Univ. de Chile

(Chile); P. Gigoux, V. Montes, F. Daruich, M. Lazo, Gemini Observatory (Chile)

7737 IT DTS: the NOAO Data Transport System [7737-68]

M. J. Fitzpatrick, National Optical Astronomy Observatory (United States)

7737 IU The Gemini Recipe System: a dynamic workflow for automated data reduction [7737-69] K. Labrie, C. Allen, P. Hirst, Gemini Observatory (United States); J. Holt, Univ. of California, Santa Cruz (United States) and Gemini Observatory (United States); R. Allen, K. Dement, Gemini Observatory (United States)

7737 IV The Spitzer Bibliography Database: bibliographic statistics [7737-70]

E. Scire, Spitzer Science Ctr., California Institute of Technology (United States); B. H. P. Chan, IPAC/NED, California Institute of Technology (United States); N. Silbermann, Spitzer Science Ctr., California Institute of Technology (United States); A. Shields, Univ. of Washington (United States) 
7737 IW Spitzer warm mission transition and operations [7737-71]

W. A. Mahoney, L. J. Garcia, Spitzer Science Ctr., California Institute of Technology (United States); J. Hunt, Jr., Jet Propulsion Lab. (United States); D. B. McElroy, V. Mannings, Spitzer Science Ctr., California Institute of Technology (United States); D. S. Mittman, Jet Propulsion Lab. (United States); J. C. O'Linger, Spitzer Science Ctr., California Institute of Technology (United States); M. Sarrel, Jet Propulsion Lab. (United States); E. Scire, Spitzer Science Ctr., California Institute of Technology (United States)

7737 1Y Toward a green observatory [7737-73]

U. Weilenmann, C. Ramírez, P. Vanderheyden, European Southern Observatory (Chile)

773712 First year of ALMA site software deployment: where everything comes together [7737-74] V. González, M. Mora, R. Araya, D. Arredondo, M. Bartsch, P. Burgos, J. Ibsen, J. Reveco, N. Sáez, A. Schemrl, J. Sepulveda, T.-C. Shen, R. Soto, N. Troncoso, M. Zambrano, Joint Atacama Large Millimeter Array Observatory (Chile); N. Barriga, Univ. Técnica Federico Santa María (Chile); B. Glendenning, National Radio Astronomy Observatory (United States); G. Raffi, European Southern Observatory (Germany); J. Kern, National Radio Astronomy Observatory (United States)

773720 Software operations support at Gemini Observatory [7737-75]

A. W. Ebbers, Gemini Observatory (United States); C. Urrutia, Gemini Observatory (Chile); T. Cumming, Gemini Observatory (United States); P. Gigoux, Gemini Observatory (Chile)

773722 The ESO Extremely Large Telescope dome: system engineering strategies for electrical power management [7737-78]

G. Marchiori, L. Giacomel, C. Manfrin, European Industrial Engineering s.r.l. (Italy)

773723 A portable observatory for persistent monitoring of the night sky [7737-79]

J. Wren, W. T. Vestrand, P. Wozniak, H. Davis, Los Alamos National Lab. (United States)

773724 From Chile to Europe in minutes: handling the data stream from ESO's Paranal Observatory [7737-81]

M. Romaniello, S. Zampieri, European Southern Observatory (Germany); C. Cerón, A. Wright, European Southern Observatory (Chile); R. Hanuschik, European Southern Observatory (Germany); C. Ledoux, European Southern Observatory (Chile); F. Comerón, European Southern Observatory (Germany)

773725 PySALT: the SALT science pipeline [7737-82]

S. M. Crawford, South African Astronomical Observatory (South Africa) and Southern African Large Telescope (South Africa); M. Still, NASA Ames Research Ctr. (United States); P. Schellart, South African Astronomical Observatory (South Africa) and Radboud Univ. (Netherlands); L. Balona, South African Astronomical Observatory (South Africa); D. A. H. Buckley, South African Astronomical Observatory (South Africa) and Southern African Large Telescope (South Africa); G. Dugmore, South African Astronomical Observatory (South Africa); A. A. S. Gulbis, A. Kniazev, South African Astronomical Observatory (South Africa) and Southern African Large Telescope (South Africa); M. Kotze, South African Astronomical Observatory (South Africa) and Univ. of Cape Town (South Africa); N. Loaring, South African Astronomical Observatory (South Africa) and Southern African Large Telescope (South Africa); K. H. Nordsieck, Univ. of Wisconsin-Madison (United States); T. E. Pickering, South African Astronomical Observatory (South Africa) and Southern African Large Telescope (South Africa); S. Potter, South African Astronomical Observatory 
(South Africa); E. Romero Colmenero, P. Vaisanen, South African Astronomical Observatory (South Africa) and Southern African Large Telescope (South Africa); T. Williams, Rutgers Univ. (United States); E. Zietsman, South African Astronomical Observatory (South Africa) and Univ. of South Africa (South Africa)

773726 Autonomous operations in extreme environments: the AMICA case [7737-83]

G. Di Rico, M. Ragni, M. Dolci, O. Straniero, A. Valentini, G. Valentini, A. Di Cianno,

C. Giuliani, INAF, Osservatorio Astronomico di Teramo (Italy); C. Bonoli, F. Bortoletto,

M. D'Alessandro, D. Magrin, INAF, Osservatorio Astronomico di Padova (Italy); L. Corcione,

A. Riva, INAF, Osservatorio Astronomico di Torino (Italy); C. Abia, Univ. de Granada (Spain);

A. Mancini, M. Busso, G. Tosti, Univ. degli Studi di Perugia (Italy)

773728 The X-shooter pipeline [7737-85]

A. Modigliani, European Southern Observatory (Germany); P. Goldoni, Lab. AstroParticule et Cosmologie (France) and DSM/IRFU/Service D'Astrophysique, CEA-Saclay (France);

F. Royer, R. Haigron, GEPI, Observatoire de Paris à Meudon (France); L. Guglielmi, Lab. AstroParticule et Cosmologie (France); P. François, GEPI, Observatoire de Paris à Meudon (France); M. Horrobin, Univ. zu Köln (Germany); P. Bristow, J. Vernet, S. Moehler, F. Kerber, P. Ballester, European Southern Observatory (Germany); E. Mason, European Southern Observatory (Chile); L. Christensen, Excellence Cluster Universe, Univ. München (Germany)

773729 The new FORS pipeline [7737-86]

C. Izzo, European Southern Observatory (Germany); L. de Bilbao, European Southern Observatory (Germany), Instituto de Física de Cantabria, CSIC (Spain), and Fundación Española para la Ciencia y la Tecnología, (Spain); J. Larsen, European Southern Observatory (Germany); S. Bagnulo, Armagh Observatory (United Kingdom); W. Freudling, S. Moehler, P. Ballester, European Southern Observatory (Germany)

7737 2A Spectroradiometric calibration of telescopes using laser illumination of flat field screens [7737-87]

J. T. Woodward, S. W. Brown, K. R. Lykke, A. W. Smith, C. E. Cramer, National Institute of Standards and Technology (United States); P. Doherty, E. Falco, C. Stubbs HarvardSmithsonian Ctr. for Astrophysics(United States)

7737 2B Changes and improvements to the Gemini North Aircraft Avoidance Program at the Gemini North Laser Guide Star facility on Mauna Kea [7737-88]

J. Archambeau, R. Oram, M. Sheehan, Gemini Observatory (United States)

7737 2D High-precision photometry with WIRCam at the CFHT [7737-90]

D. Devost, L. Albert, D. Teeple, Canada-France-Hawaii Telescope Corp. (United States);

B. Croll, Univ. of Toronto (Canada)

7737 2F Characterization of the mid-IR image quality at Gemini South [7737-92]

D. Li, C. M. Telesco, F. Varosi, Univ. of Florida (United States)

Author Index 


\title{
Conference Committee
}

\author{
Symposium Chairs
}

Masanori lye, National Astronomical Observatory of Japan (Japan)

Douglas A. Simons, Gemini Observatory (United States)

Symposium Cochairs

Mark M. Casali, ESO-European Organisation for Astronomical research in the Southern Hemisphere (Germany)

Kathryn A. Flanagan, Space Telescope Science Institute (United States)

Conference Chairs

David R. Silva, National Optical Astronomy Observatory (United States)

Alison B. Peck, Atacama Large Millimeter/Submillimeter Array (Chile)

B. Thomas Soifer, California Institute of Technology (United States)

Program Committee

David S. Adler, Space Telescope Science Institute (United States)

Roger J. Brissenden, Chandra Science Center, Harvard-Smithsonian Center for Astrophysics (United States)

Dennis R. Crabtree, Gemini Observatory (Chile) and Hertzberg Institute of Astrophysics (Canada)

Suzanne R. Dodd, Spitzer Science Center/California Institute of Technology (United States)

Richard F. Green, Large Binocular Telescope Observatory, The University of Arizona (United States)

Andreas Kaufer, European Southern Observatory (Chile)

Nicole M. Radziwill, James Madison University (United States) and National Radio Astronomy Observatory (United States)

Robert L. Seaman, National Optical Astronomy Observatory (United States)

R. Christopher Smith, National Optical Astronomy Observatory (United States)

Session Chairs

1 Site and Facility Operations I

Andreas Kaufer, European Southern Observatory (Chile) 
2 Site and Facility Operations II

Richard F. Green, Large Binocular Telescope Observatory, The University of Arizona (United States)

3 Science Operations Processes

Richard F. Green, Large Binocular Telescope Observatory, The University of Arizona (United States)

4 Time Domain and Target of Opportunity

Nicole M. Radziwill, James Madison University (United States) and National Radio Astronomy Observatory (United States)

5 Transient Events and Observatory Operations

Robert L. Seaman, National Optical Astronomy Observatory (United States)

6 Dynamic Observatory Scheduling

Robert L. Seaman, National Optical Astronomy Observatory (United States)

$7 \quad$ Remote Robotic and Service

David R. Silva, National Optical Astronomy Observatory (United States)

$8 \quad$ Archive Operations and Legacy

Suzanne R. Dodd, Spitzer Science Center/California Institute of Technology (United States)

9 User Support

David S. Adler, Space Telescope Science Institute (United States)

10 Operations and Data Quality Control

David S. Adler, Space Telescope Science Institute (United States) 